\title{
Influence of elastic strains on LPE growth kinetics in the $\mathrm{Cd}-\mathrm{Hg}-\mathrm{Te}$ system
}

\author{
P.P. Moskvin ${ }^{1}$, L.V. Rashkovetsky ${ }^{2}$, V.V. Khodakovsky ${ }^{1}$

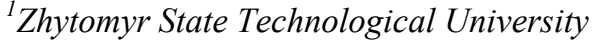 \\ 103, Chernyakhovsky str., 10005 Zhytomyr, Ukraine \\ E-mail:moskvin@us.ztu.edu.ua \\ ${ }^{2}$ V. Lashkaryov Institute of Semiconductor Physics, NAS of Ukraine \\ 45, prospect Nauky, 03028 Kyiv, Ukraine
}

\begin{abstract}
By comparing the results of calculations concerning the dependence of the parameters of a layer on the growth conditions with and without regard for mechanical strains in the growing system, we have analyzed the influence of the elastic energy of the strained solid phase on the phase formation in the $\mathrm{Cd}-\mathrm{Hg}-\mathrm{Te}$ system. It is shown that the occurrence of elastic strains in a layer results in an insignificant reduction of the growth rate and has almost no influence on the composition of a growing layer. The ideas of coherently matched phases in the presence of elastic deformations in the system, as well as the assumption about the existence of the chemical equilibrium of phases on the interface, give rather close results as for the crystallization of the material. Both approaches describe the experimental data on the growth of layers in various temperature-time regimes quite satisfactorily.
\end{abstract}

Keywords: $\mathrm{A}^{2} \mathrm{~B}^{6}$ semiconductors, LPE, elastic strains.

Manuscript received 12.07.07; accepted for publication 27.09.07; published online 30.11.07.

$\mathrm{Cd}-\mathrm{Hg}-\mathrm{Te}$ systems including solid-solution layers with thickness of several micrometers are widely used as materials for infrared detectors. They are obtained by the growing from the own melt on the substrate made of a binary compound (mostly it is cadmium telluride). In works $[1,2]$, the thermodynamic information allowing one to analyze the system's phase diagram is theoretically obtained with high reliability. In [3, 4], the influence of the growth kinetics on the composition of layers is considered in terms of a diffusion-limited model. According to this model, the thermodynamic equilibrium was supposed to exist on the interface. Thus, the equations of phase equilibrium $[1,2]$ are valid for the concentrations of components on a growing surface, and the diffusion mass transfer problem can be posed. At the same time, the difference (although insignificant) between the crystal lattice constants of initial mixed compounds, CdTe and $\mathrm{HgTe}$, occurs in the $\mathrm{Cd}-\mathrm{Hg}-\mathrm{Te}$ system. During the solid solution crystallization on the substrate from a binary compound, this factor is responsible for the occurrence of elastic strain fields in the growing system. Just this excess mixing energy component responds for the formation of misfit dislocations near the "substrate-layer" interface and it, probably, governs complex diffusion phenomena in the solid phase, by determining the shape of a heterojunction.

Thus, it is possible to consider the grown epitaxial layer to be elastically strained, and the description of such crystallization process should involve the presence of the elastic energy in the system. This means that, in the description of the material growth kinetics, the boundary concentrations of components on the "strained solid phase-melt" interface should be connected by the coherent diagram equations $[5,6]$, instead of equations which take into account only the chemical phase equilibrium.

Therefore, the purpose of the present work is to analyze the influence of a shift of phase equilibria due to the absent or incomplete relaxation of elastic strains in $\mathrm{Cd}_{\mathrm{x}} \mathrm{Hg}_{1-\mathrm{x}} \mathrm{Te}$ solid solutions on the heteroepitaxy kinetics for a layer grown from binary compounds on a substrate. In view of important practical applications of the posed problem, it is necessary to theoretically estimate the possibility to rule the strains in a heterojunction by changing the dependence of a composition of the layer on its thickness while obtaining the layers under nonisothermal conditions. 
The equations of phase equilibria between the elastically strained solid phase and the own melt for ternary systems are obtained in $[5,6]$. On their derivation, the thermodynamic functions describing the liquid phase were supposed to be the same as those in the description of general phase equilibria, whereas the calculation of component activities in the solid state was performed, by considering the existence of elastic strain fields occurring in a thin layer of the solid solution coherently matched with a massive substrate. For the $\mathrm{Cd}-\mathrm{Hg}-\mathrm{Te}$ system, these equations look as

$$
\begin{aligned}
& \Delta S_{\mathrm{CdTe}}^{F}\left(T_{\mathrm{CdTe}}^{F}-T\right) / R T+ \\
& +\Delta C_{\mathrm{Cd}-\mathrm{Te}}^{F}\left[\left(T-T_{\mathrm{CdTe}}^{F}\right)-T \ln \frac{T}{T_{\mathrm{CdTe}}^{F}}\right] / R T+\ln \frac{x_{\mathrm{Cd}} \cdot x_{\mathrm{Te}}}{x_{\mathrm{Cd}}^{S t} \cdot x_{\mathrm{Te}}^{S t}}= \\
& =\ln \gamma_{\mathrm{CdTe}} x_{\mathrm{CdTe}}+B \cdot\left[\left(1-x_{S}\right)^{2}-(1-x)^{2}\right] / R T ; \\
& \Delta S_{\mathrm{HgTe}}^{F}\left(T_{\mathrm{HgTe}}^{F}-T\right) / R T+\Delta C_{\mathrm{Hg}-\mathrm{Te}}^{F} \times \\
& \times\left[\left(T-T_{\mathrm{HgTe}}^{F}\right)-T \ln \frac{T}{T_{\mathrm{HgTe}}^{F}}\right] / R T+\ln \frac{x_{\mathrm{Hg}} \cdot x_{\mathrm{Te}}}{x_{\mathrm{Hg}}^{S t} \cdot x_{\mathrm{Te}}^{S t}}= \\
& =\ln \gamma_{\mathrm{HgTe}} x_{\mathrm{HgTe}}+B \cdot\left(x_{S}^{2}-x^{2}\right) / R T ; \\
& \Delta C_{\mathrm{A}-\mathrm{Te}}^{F}=C_{\mathrm{A}}^{L}+C_{\mathrm{Te}}^{L}-C_{\mathrm{ATe}} ; R T \cdot \ln \gamma_{j}=\alpha^{S}\left(1-x_{j}\right)^{2} ; \\
& x_{\mathrm{HgTe}}+x_{\mathrm{CdTe}}=1 ; \mathrm{A}=\mathrm{Cd}, \mathrm{Hg} ; j=\mathrm{CdTe}, \mathrm{HgTe},
\end{aligned}
$$

where $T^{F}, \Delta S^{F}$ - melting temperature and entropy of fusion of CdTe, HgTe compounds. "St" stands for the stoichiometric liquid phase, $C_{i}-$ molar heat capacities of the initial elements of the liquid phase and ATe compounds; $\gamma_{j}, \alpha^{S}$ - the activity coefficient of the $j$-th component and the solid phase interaction parameter; $x_{j}, x_{S}-$ compositions of the solid solution and substrate; and $B$ - elastic constant of the solid solution dependent on the crystallographic orientation of a layer.

It is known $[5,6]$ that the value of parameter $B$ for a (111)-oriented layer is a little more than the respective value for a (100)-oriented one. Taking into account that, in practice, (111)-oriented layers are grown more often and considering that, as a whole, the elastic energy contribution to the total energy balance of the solid phase is expected to be not so significant, the following calculations are carried out just for the latter layer orientation. Then, according to the data $[5,6]$ for $(111)$ oriented layers of crystals with cubic symmetry, this parameter is the following combination of elastic constants:

$B=\frac{N_{\mathrm{A}}}{4}\left(\frac{d a}{d x}\right)^{2} a \frac{6\left(c_{11}+2 c_{12}\right) c_{44}}{\left(c_{11}+2 c_{12}+4 c_{44}\right)}$.

Here, $a, c_{k m}$ - crystal lattice parameter of an unstrained solid solution and its elastic constants, and $N_{\text {A }}-$ Avogadro number.
Elastic constants, as well as the crystal lattice parameter for the current solid solution composition, were calculated by the method of linear interpolation, by using the data on the appropriate properties of initial binary compounds as the initial information. Data necessary for the calculation of the mentioned parameters are borrowed from work [7] and submitted in Table.

Table. Elastic constants of CdTe and HgTe compounds at various temperatures [7].

\begin{tabular}{|l|l|l|l|l|}
\hline Compound & $T, \mathrm{~K}$ & $\begin{array}{l}C_{11}, \\
\mathrm{GPa}\end{array}$ & $\begin{array}{c}C_{12}, \\
\mathrm{GPa}\end{array}$ & $\begin{array}{l}C_{44}, \\
\mathrm{GPa}\end{array}$ \\
\hline \multirow{3}{*}{$\mathrm{CdTe}$} & 298 & 53.8 & 37.4 & 20.18 \\
& & & & \\
\cline { 2 - 5 } & 77 & 56.2 & 39.4 & 20.61 \\
\hline \multirow{2}{*}{$\mathrm{HgTe}$} & 300 & 53.6 & 33.6 & 22.30 \\
& & & & \\
\cline { 2 - 5 } & 77 & 58.7 & 40.5 & 21.10 \\
\hline
\end{tabular}

As follows from Table, the elastic constants of compounds are dependent on temperature.

While the experimental data concerning these parameters are absent for the temperatures of growth, their values were corrected by diminution by $10 \%$ relatively to respective ones for room temperature. Such a degree of correction corresponded to their reduction up to values at the typical temperatures of crystallization in the system.

The thermodynamic functions needed to calculate the liquid phase parameters within the model of polyassociated solutions are taken from our works $[1,2]$.

The shift of phase equilibria due to the presence of elastic strains in the multicomponent system is characterized by the contact supercooling which is defined as the difference between the liquidus temperature and the temperature of phase equilibrium between the strained solid phase and the melt of the same composition $[5,6]$. The calculated values of contact supercooling on the solid solution growth on CdTe and HgTe (111)-oriented substrates, which are obtained by solving Eqs. (1), are shown in Fig. 1. As follows from these data, the contact supercooling for typical growth temperatures is not greater than $1 \mathrm{~K}$. Such a result is not unexpected, taking into account the abovementioned properties of the solid solutions under study. Really, the direct calculations of the elastic component in Eq. (1) show that the contribution of external elastic strains into the solid-phase total energy is not greater than $10 \%$ relative to the excess mixing energy of the solid phase (parameter $\alpha^{S}$ ). The last data concern the (111)-oriented layer growth. For (100)-oriented layers, the calculated contact supercooling is even less.

The occurrence of elastic energy in the growing system results not only in a change of the phase equilibrium temperature, but also in a change of the 


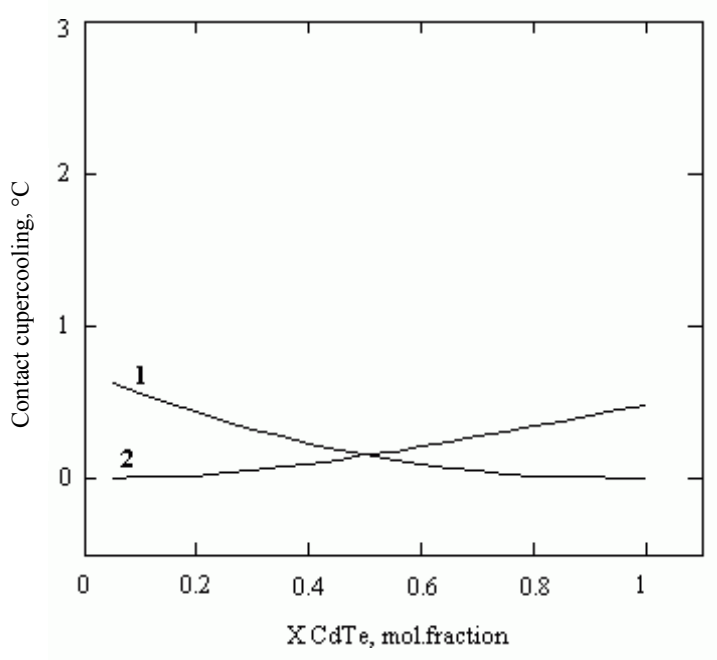

Fig. 1. Contact supercooling with solid solution composition at the liquidus temperature $T_{L}=550^{\circ} \mathrm{C}$ for $\mathrm{CdTe}(1)$ and HgTe (2) (111)-oriented substrates.

composition of the growing strained solid phase. This effect is known as the lattice-pulling effect [5]. The calculations of this effect with the use of the equations of the coherent diagram (1) have shown that the compositions of strained and equilibrium solid phases differ only in the third digit after a point, i.e. the considered effect is rather insignificant. Thus, the obtained data allow us to assume that the mechanical strains in a growing solid solution should result in an insignificant diminution of the growth rate of layers due to the contact supercooling at an insignificant change of the growing material's composition relative to the composition predicted by the equilibrium phase diagram. Such a distinction in the characteristic curves of both coherent and equilibrium $\mathrm{Cd}-\mathrm{Hg}-\mathrm{Te}$ system's phase diagrams should be taken into account in the growth kinetics analysis.

To describe the growth kinetics of a ternary solution strained layer, we should solve the system of differential equations of diffusion. The assumption about a coherent match between the growing layer and the substrate should be taken as boundary conditions for them, instead of the assumption about the chemical equilibrium on the interface. In such a situation, the coherent diagram equations (1) are valid for the concentrations of components on the interface, while the equations of mass conservation remain constant $[5,8]$. Thus, the mathematical description of the process of growth of the coherently matched epilayer and a massive substrate is given by the equations

$$
\begin{aligned}
& \frac{\partial x_{i}}{\partial t}=D_{i} \frac{\partial^{2} x_{i}}{\partial z^{2}}+\frac{\rho_{s}}{\rho} V \frac{\partial x_{i}}{\partial z}, \\
& \frac{\rho_{s}}{\rho_{l}} V\left(x_{S, i}^{0}-x_{i}^{0}\right)=D_{i} \frac{\partial x_{i}(0, t)}{\partial z}
\end{aligned}
$$

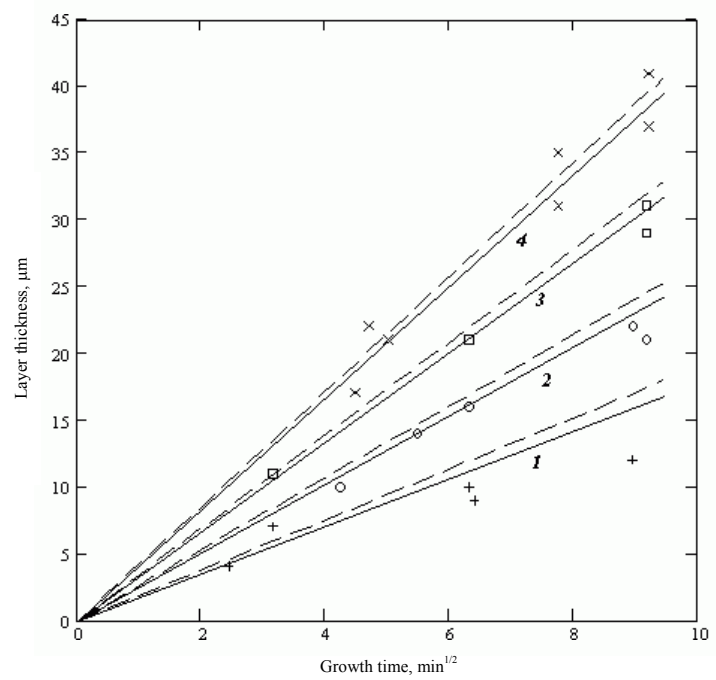

Fig. 2. Layer thickness with square root of time for $\mathrm{Cd}_{\mathrm{x}} \mathrm{Hg}_{1-\mathrm{x}} \mathrm{Te}$ solid solution isothermal growth with $x \approx 2$ mol. frac. without elastic strains taken into account (dashed curve) and with them (solid curve). Growth temperatures: $1-501 ; 2-503 ; 3-505$, $4-507^{\circ} \mathrm{C}$. Liquidus temperature $T_{L}=513^{\circ} \mathrm{C}$.

where $x_{s, i}^{0}, x_{i}^{0}-$ concentrations of solid and liquid phase components on the interface, respectively, corresponding to the coherent diagram equations (1), $D_{i} \frac{\partial x_{i}}{\partial z}-$ component fluxes nearby the surface boundary to the interface; $V(t)$ - growth rate; $\frac{\rho_{s}}{\rho_{l}}-$ ratio of the solid- and liquid-phase molar densities; $i=$ $\mathrm{Cd}, \mathrm{Hg}$ - under the solid solution growth from a tellurium enriched liquid phase, $z$ - coordinate directed deep into the melt.

The mass transfer problem formulated in the mentioned way was solved by numerical methods both for isothermal growth conditions and for the layers grown by the equilibrium and supercooling techniques. In the last case, the process of growth was realized by the uniform cooling of a liquid phase supercooled by $\Delta T$. So Fig. 2 shows the calculated and experimental data [9] on the solid-solution layer thickness grown on the CdTe (111)B substrate with isothermal growth time for different supercoolings and with mechanical strains, being taken into account or not. The partial diffusion coefficients necessary for the calculations were supposed to be independent of temperature in the considered interval and equal to: $D_{\mathrm{Hg}}=2.5 \cdot 10^{-5} \mathrm{~cm}^{2} / \mathrm{s}$ and $D_{\mathrm{Cd}}=2.8 \cdot 10^{-5} \mathrm{~cm}^{2} / \mathrm{s}$ [9]. As follows from the calculations, elastic strains and the contact supercooling in the system reduce the total liquid phase supercooling degree and slow down the material's growth rate, which is reflected in a reduction of the layer thickness. However, in general, the influence of contact supercooling on the crystallization process is not significant. This means that the kinetic calculations carried out by 
using the phase equilibrium relations and the coherent ones as boundary conditions, give close results, and both these approaches describe the isothermal crystallization quite satisfactorily.

Influence of mechanical strains must be the most essential while growing the layers of a variable composition. Layer composition changes in such a situation occur, first of all, due to a temperature decrease in the system. For the quantitative description of the layer growth at a temperature decrease in the system, the problem of diffusion was solved in such a way that, on each iterative step of integration, the growth temperature was reduced linearly with the given rate. The growth rate of a layer, its composition, and thickness at each time moment were obtained. The comparison of calculated and experimental results [10] for the thickness of layers grown by the supercooling technique at various degrees of supercooling and cooling rates is given in Fig. 3. Quite satisfactory conformity of the results of calculations to experimental data is observed. Such a result confirms the efficiency of the diffusion-limited model in the description of the solid solution LPE process, when the model of polyassociative solutions is used for the description of a phase equilibrium in the system.

To estimate the influence of mechanical strains on the growth process, the comparative analysis of the results of calculations of the solid solution composition distribution in the cases where layers are grown under various time-temperature conditions both with and without the account of elastic energy in the total system's energy balance should be carried out. Such data

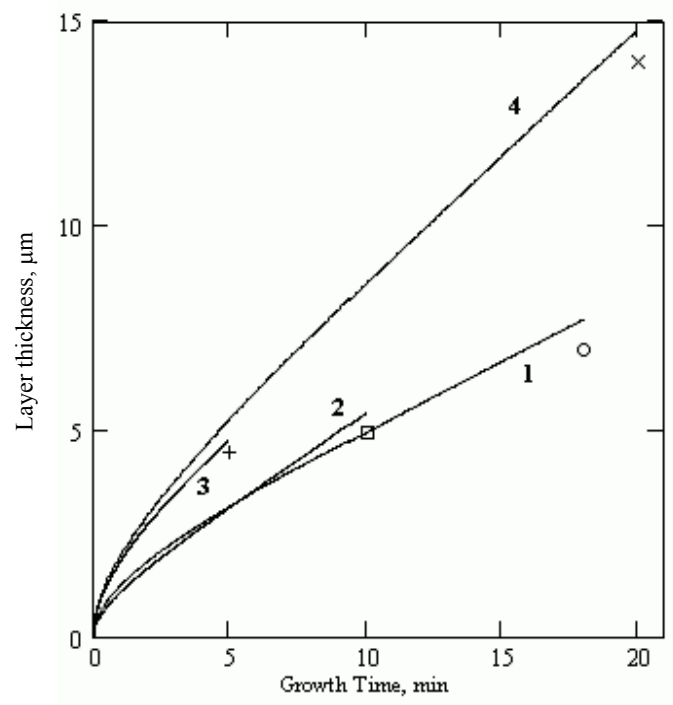

Fig. 3. $\mathrm{Cd}_{\mathrm{x}} \mathrm{Hg}_{1-\mathrm{x}}$ Te layer thickness with $x \approx 0.8 \mathrm{~mol}$. frac. versus growth time. Liquidus temperature $T_{1}=462^{\circ} \mathrm{C} .1$ initial growth temperature $T=455^{\circ} \mathrm{C}\left(\Delta T=7{ }^{\circ} \mathrm{C}\right)$, cooling rate $V_{c}=0.25^{\circ} \mathrm{C} / \mathrm{min} ; 2-T=456^{\circ} \mathrm{C}\left(\Delta T=8^{\circ} \mathrm{C}\right), V_{c}=$ $0.54^{\circ} \mathrm{C} / \mathrm{min}, 3-T=452^{\circ} \mathrm{C}\left(\Delta T=10^{\circ} \mathrm{C}\right), V_{c}=0.56^{\circ} \mathrm{C} / \mathrm{min}$, $4-T=450^{\circ} \mathrm{C},\left(\Delta T=12^{\circ} \mathrm{C}\right), V_{c}=0.53^{\circ} \mathrm{C} / \mathrm{min}$. Experimental data [14].

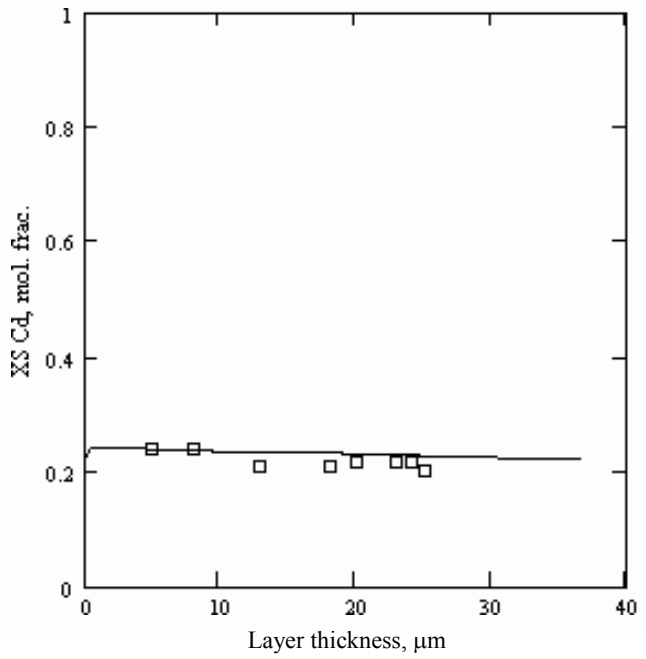

Fig. 4. Solid solution composition versus layer thickness. Initial growth temperature $T=552^{\circ} \mathrm{C}$. Liquidus temperature $T_{1}=$ $=555^{\circ} \mathrm{C}$. Cooling rate $V_{c}=0.25^{\circ} \mathrm{C} / \mathrm{min}$. Experimental data [11].

for a solid solution grown from the liquid phase with the composition $x_{\mathrm{Cd}}=0.006$ at. frac., $x_{\mathrm{Hg}}=0.217$ at. frac. at an initial supercooling of $2{ }^{\circ} \mathrm{C}$ and various cooling rates are shown in Fig. 5. It is obvious from Fig. 5 that the results of calculations of the composition distribution carried out with and without account of mechanical strains differ poorly. The calculated dependences carried out with the coherent diagram application give some reduction of a composition gradient relatively to those calculated with the use of the phase equilibrium conditions. Naturally, these data reflect the well-known effect of solid solution composition pulling to a substrate's one (lattice-pulling effect) [6]. However, in case of $\mathrm{Cd}_{\mathrm{x}} \mathrm{Hg}_{1-\mathrm{x}} \mathrm{Te}$ solid solution, for which the variation of a lattice constant with the composition change is very insignificant, this effect is noticed to be poor. We note that, with decrease in the initial growth temperature, the considered effect should be manifested less significantly. It is illustrated by the calculated data in Fig. 5 when the initial growth temperature is reduced down to $450^{\circ} \mathrm{C}$.

It is worth to note the observed satisfactory correlation between the calculated values of composition gradients over the layer's thickness and the experimental data [13] and a little worse correspondence for the solid phase composition.

We note that, under the typical growth conditions of an epitaxial layer in the considered system, the experimental data on the composition variation versus the layer thickness are close to linear. This means that, in the considered temperature intervals, the liquidus and solidus slopes of the system can be plotted as straight lines with high degree of accuracy, i.e., the linear model of diffusion growth earlier developed for the description of a similar process in systems of $\mathrm{A}^{3} \mathrm{~B}^{5}$ solid solutions [6] can be also applied to this system of semiconductor 


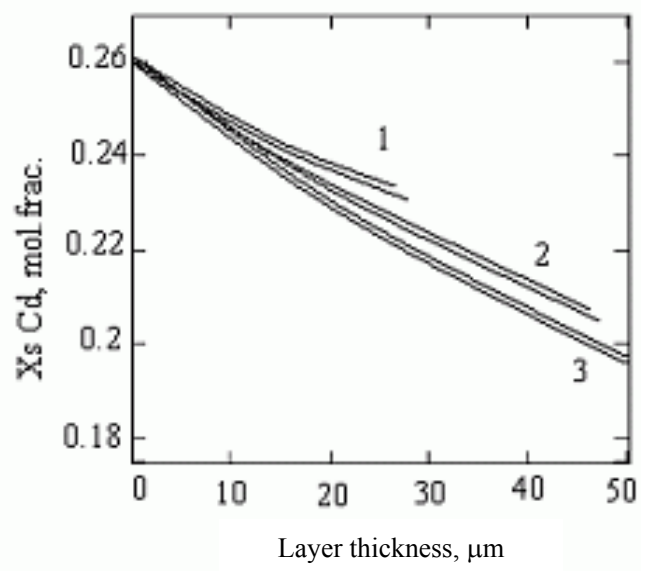

Fig. 5. $\mathrm{Cd}_{\mathrm{x}} \mathrm{Hg}_{1-\mathrm{x}} \mathrm{Te}$ solid solution composition versus layer thickness for the initial growth temperature $T=500{ }^{\circ} \mathrm{C}$, degree of supercooling $\Delta T=2{ }^{\circ} \mathrm{C}$, different cooling rates, and elastic strains taken or not into account: $V_{c}=0.2^{\circ} \mathrm{C} / \mathrm{min}(1)$, $0.4(2), 0.6(3)$.

solid solutions. It is also necessary to note that the quantitative application of the diffusion-limited model with linear forms of the phase diagram surface will result inevitably in mathematical difficulties in calculations of the model's characteristic parameters (for example, the partial liquidus slopes $\partial x_{i} / \partial T$ ). The model's software offered in this work has no such lacks.

Thus, the achieved good correspondence of the results of calculations of the growth kinetics of $\mathrm{Cd}_{\mathrm{x}} \mathrm{Hg}_{1-\mathrm{x}} \mathrm{Te}$ solid solutions in various time-temperature regimes to experiments confirms the applicability of the diffusion-limited model to the description of the growth process of layers. The solution of the problem of growth under mechanical strains in the system shows that the elastic energy reduces the material's growth rate by $10 \%$ on the average. Thus, a variation in the composition of an elastically strained layer is insignificant and not greater than several percents in comparison with that predicted by the equilibrium phase diagram.

\section{References}

1. P.P. Moskvin, L.V. Rashkovets'kyi // Zhurnal Fiz. Khimii 80(9), p. 1568-1573 (2006) (in Russian).

2. P.P. Moskvin, V.V. Khodakovsky, L.V. Rashkovets'kyi // Semiconductor Physics, Quantum Electronics \& Optoelectronics 9(3), p. 12-16 (2006).

3. P.P. Moskvin, V.V. Khodakovsky // Proc. of Confer. "FAGRAN-2006", Voronezh, 1, p. 380-383 (2006).

4. P.P. Moskvin, V.V. Khodakovsky // Proc. of Confer. "SEMST-2", Odessa, p. 122 (2006).

5. V.V. Kuznetsov, P.P. Moskvin, V.S. Sorokin // J. Crystal Growth 66(3), p. 562-575 (1984).

6. V.V. Kuznetsov, P.P. Moskvin, V.S. Sorokin, Nonequilibrium Phenomenon at Liquid Heteroepitaxy of Semiconductor Solid Solutions. Metallurgiya, Moscow, 1991 (in Russian).

7. R.D. Greenough, S.B. Palmer // J. Phys. D6, p. 587 (1973).

8. M.B. Small, R. Ghez // J. Appl. Phys. 50(8), 5322 (1979).

9. P.P. Moskvin, V.V. Khodakovsky, L.V. Rashkovets'kyi // Proc. of Confer. "METIT-3", Kremenchug, p. 2 (2006).

10. R.A. Wood, R.J. Hager // Vac. Sci. Technol. A1(3), p. 1806-1810 (1983).

11. J. A. Mroczkowski, H. R. Vydyanath // J. Elektrochem. Soc. 128(3), p. 655-661 (1981).

12. C.C. Wang, S.H. Shin M. Chu, M. Lanir, A.H.B. Vanderwyck // J. Electrochem. Soc. 127(1), p. 175-179 (1980).

13. J.E. Bowers, J.L. Schmit, C.J. Speerschneider, R.B. Maciolek // IEEE Trans. Electron. Devices, ED27(1), p. 25 (1980).

14. A. Lusson, R. Triboulet // J. Crystal Growth, 85, p. 503-509 (1987). 